\title{
Tu vas à la cerise les dimanches? L'importance de travailler la perception et la production de phonèmes en allemand langue étrangère
}

\author{
Isabelle Wouters \\ Université du Québec à Montréal \\ Véronique Fortier \\ Université du Québec à Montréal
}

\begin{abstract}
Résumé
L'enseignement de la prononciation est loin d'avoir été aussi étudié que, par exemple, l'enseignement de la grammaire et du vocabulaire (Saito, 2015), et les participants des études sur le sujet ont le plus souvent l'anglais comme langue maternelle ou langue cible (Lee, Jang, et Plonsky, 2015). Ainsi, afin d'enrichir la recherche dans ce domaine et d'en étendre la portée à d'autres langues, nous avons mené une étude exploratoire basée sur un devis quasi expérimental auprès de 64 participants francophones apprenant l'allemand comme langue étrangère. Nous nous sommes penchées sur cinq sons consonantiques jugés problématiques pour les francophones, visés lors de cinq courtes séquences d'enseignement explicite offertes à deux groupes expérimentaux. Les résultats des participants aux tests (perception et production) faits avant et après les séquences expérimentales montrent que l'enseignement proposé, bien que de courte durée (total : 65 minutes), a permis aux groupes expérimentaux de montrer des gains significatifs.
\end{abstract}

\begin{abstract}
Pronunciation instruction has been the focus of relatively few studies compared to grammar and vocabulary instruction (Saito, 2015), and generally, studies involving pronunciation instruction have had English as the participants' first or target language (Lee, Jang, \& Plonsky, 2015). Thus, we set out to investigate pronunciation instruction and to extend the scope of study to other languages in an exploratory study based on a quasi-experimental design with 64 French-speaking participants learning German as a foreign language. We focused on five consonant sounds considered challenging for French speakers, which were targeted during five short explicit teaching sequences given to two experimental groups. Participants' results in perception and production tasks before and after the experimental sequences show that the suggested teaching, although of short duration (total time: 65 minutes), led experimental groups to show significant gains.
\end{abstract}




\section{Tu vas à la cerise les dimanches ? L'importance de travailler la perception et la production de phonèmes en allemand langue étrangère}

Malgré l'intérêt récent démontré par les chercheurs envers l'enseignement de la prononciation (Thomson et Derwing, 2015), cet objet d'enseignement et de recherche a longtemps été largement moins étudié que d'autres objets comme le vocabulaire ou la grammaire (Derwing et Munro, 2005; Saito, 2012, 2015). À cet effet, Foote, Holtby et Derwing (2011) ont fait ressortir que les enseignants de langues secondes (L2) ${ }^{1}$ semblaient eux-mêmes considérer l'enseignement du vocabulaire et de la grammaire comme étant plus crucial que l'enseignement de la prononciation et que, conséquemment, ils accordaient très peu de temps de classe à cette dernière $(<5 \%)$. Ce manque d'intérêt, partagé par chercheurs et enseignants, peut cependant avoir des conséquences pour l'apprenant d'une L2, car une mauvaise prononciation qui réduirait l'intelligibilité, c'est-à-dire la somme de ce qu'il est possible de comprendre d'un énoncé fait par un locuteur L2 (Derwing, Munro, et Wiebe, 1998; Thomson et Derwing, 2015), pourrait être un prétexte pour une évaluation sociale négative ou discriminatoire (Derwing et Munro, 2005). Ainsi, la nécessité de se pencher sur l'enseignement de la prononciation, soit la performance de perception et de production d'un apprenant au niveau segmental (voyelles et consonnes) et au niveau suprasegmental (prosodie, intonations, rythme, accentuation) (Kennedy, Blanchet, et Trofimovich, 2014) revêt un caractère social important.

Pour les enseignants néanmoins désireux d'enseigner la prononciation, peu de pistes concrètes d'enseignement sont proposées, obligeant ces derniers à se fier à leur intuition (Derwing et Munro, 2005). Ce manque de ressources pousse certains enseignants à orienter leurs pratiques afin de proposer l'atteinte d'un niveau de prononciation en L2 s'approchant de celui du locuteur natif (Derwing et Munro, 2005 ; Levis, 2005 ; Lee, Jang, et Plonsky, 2015), qui a été décrit par Levis (2005) comme étant le principe de nativeness. Cependant, des études en enseignement de la prononciation ont fait ressortir l'invraisemblance de viser un niveau près du locuteur natif en L2 considérant, d'une part, l'absence d'homogénéité chez les locuteurs natifs qui rend impossible l'identification d'un modèle unique et, d'autre part, les difficultés rencontrées pour atteindre un tel but notamment du côté des apprenants adultes, dont un très petit nombre arrive à atteindre un tel niveau de compétence (Derwing et coll., 1998 ; Jenkins, 2002 ; Saito et Lyster, 2012 ; Loewen, 2015 ; Lee et coll., 2015). C'est pourquoi des auteurs ont proposé de promouvoir plutôt dans l'enseignement la nécessité pour les apprenants d'être compris lorsqu'ils s'expriment en L2 (Levis, 2005 ; Derwing et Munro, 2005 ; Thomson et Derwing, 2015 ; Derwing, 2018), ce que sous-tend le principe d'intelligibilité de Levis (2005). Ce principe stipule que l'enseignement de la prononciation devrait viser l'augmentation de l'intelligibilité, la somme de ce qui est compris d'un énoncé (Derwing et coll., 1998) ou de la compréhensibilité, soit la facilité avec laquelle un apprenant L2 est compris (Derwing et coll., 1998).

Ainsi, en orientant plutôt les pratiques enseignantes vers le développement de l'intelligibilité ou de la compréhensibilité des apprenants, les chercheurs recommandent de travailler différentes composantes de la prononciation (Derwing et coll., 1998 ; Derwing et Munro, 2005 ; Lee et coll., 2015). En effet, des auteurs (p.ex., Derwing et coll., 1998 ; Munro et Derwing, 2006 ; Jenkins 2002 ; Hahn, 2004 ; Field, 2005 ; Cardoso, 2018) ont constaté qu'un travail des composantes segmentales (associées aux consonnes et aux voyelles) et suprasegmentales (associées à la syllabe, l'intonation et le rythme) de la prononciation permettrait d'augmenter de différentes façons et à des degrés différents l'intelligibilité ou la 
compréhensibilité des apprenants. À cet effet, Derwing et coll. (1998) ont mené une étude auprès de trois groupes d'adultes de niveau intermédiaire en anglais L2 afin de vérifier les effets d'un enseignement visant une des deux composantes de la prononciation sur l'accent, la compréhensibilité et la fluidité. Un des deux groupes expérimentaux a reçu un enseignement au niveau segmental de la prononciation, le second, au niveau suprasegmental et le groupe témoin n'a pas reçu d'enseignement spécifique. Les résultats ont suggéré que l'enseignement au niveau suprasegmental apportait des gains significatifs pour l'amélioration de l'accent, de la compréhensibilité et de la fluidité des participants de ce groupe. Les auteurs (Derwing et coll., 1998) ont rapporté que l'augmentation significative de la compréhensibilité des apprenants ayant reçu un enseignement au niveau suprasegmental lors du récit d'une histoire improvisée s'expliquerait par le fait que les composantes du niveau suprasegmental étaient plus saillantes dans de longs énoncés. Un travail visant spécifiquement cette composante peut alors aider les apprenants à être plus compréhensibles lorsqu'ils doivent exprimer de longues phrases. Pour ce qui est du niveau segmental, l'enseignement a aidé les participants à porter leur attention sur la forme et ainsi réduire leur accent et augmenter leur compréhensibilité dans un test contrôlé (Derwing et coll., 1998). L'enseignement au niveau segmental pourrait alors être utile lors de bris de communication portant sur une mauvaise prononciation d'un phonème (Derwing et coll., 1998). Effectivement, les participants ayant reçu l'enseignement à ce niveau de la prononciation pourraient avoir les connaissances nécessaires pour corriger un phonème mal prononcé et ainsi rendre leurs énoncés plus compréhensibles.

Cependant, l'enseignement de ces différentes composantes de la prononciation devra être adapté à certaines caractéristiques des apprenants, comme l'âge, qui influencerait la manière d'interpréter et de percevoir les sons, ainsi que leur façon d'apprendre ; nous discuterons de ces deux facteurs dans ce qui suit.

Relativement à l'âge, certains auteurs avancent que les apprenants adultes interpréteraient souvent, de façon erronée, les phonèmes d'une L2 avec les paramètres de la L1 et qu'ils auraient de la difficulté à créer de nouvelles catégories de sons pour les sons des L2 (Flege Yeni-Komshian et Liu, 1999 ; Best et Tyler, 2007 ; Baker, Trofimovich, Flege, Mack, et Halter, 2008). Néanmoins, l'apprentissage d'une L2 nécessite, au besoin, la création de nouvelles catégories de sons par les apprenants, puisque les langues n'ont pas d'emblée les mêmes phonèmes (Flege, 1995 ; Gut, 2009). Ce principe sous-tend le modèle d'apprentissage de la prononciation (Speech Learning Model) de Flege (1995), un modèle permettant de prédire la difficulté d'apprentissage des sons d'une langue cible (voir Figure 1). 


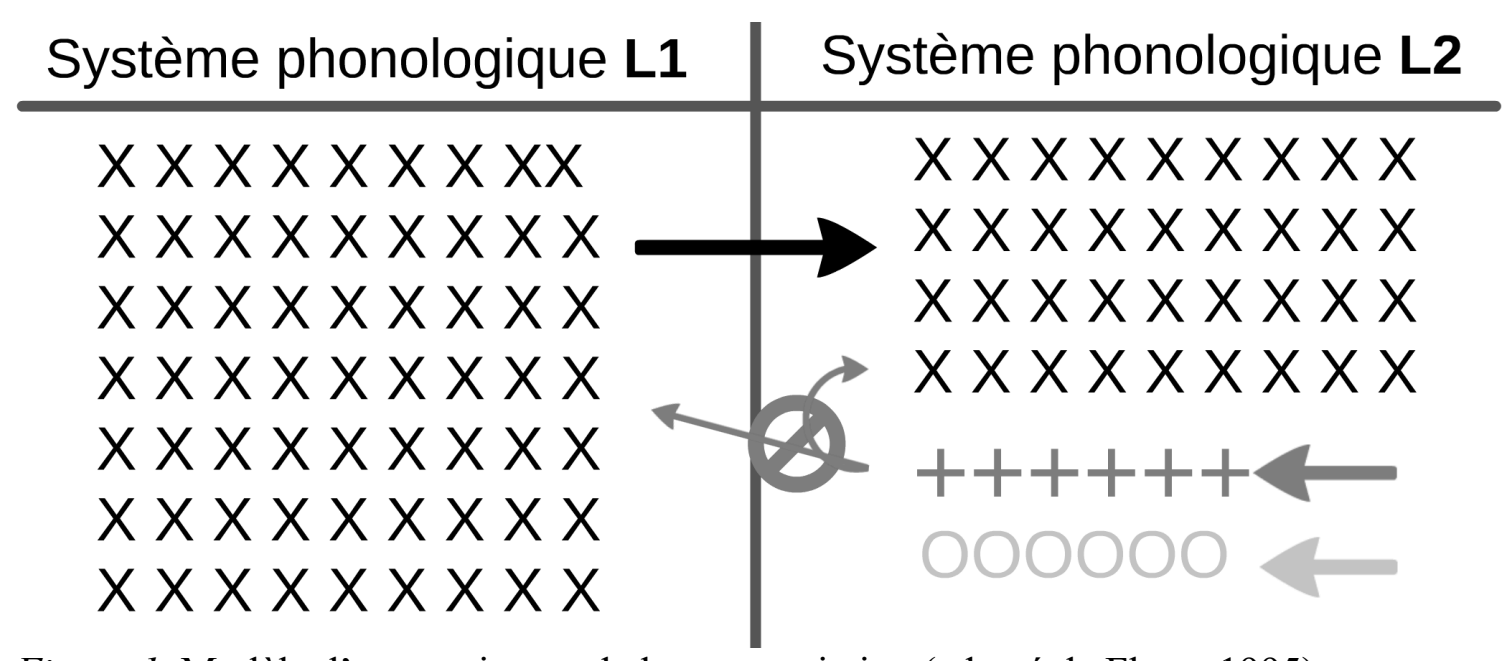

Figure 1. Modèle d'apprentissage de la prononciation (adapté de Flege, 1995).

Selon ce modèle, quelques phonèmes qui composent le système phonologique de la L1 se retrouvent également dans le système phonologique de la L2 et ne poseront donc aucune difficulté lors de l'apprentissage de la langue cible (voir les « $\mathrm{X}$ » dans la figure 1 qui représentent les catégories phonologiques de la L1 qui existent également dans le système phonologique de la L2). Également, les phonèmes complètement différents de ceux du répertoire de la L1 pourraient s'acquérir avec une certaine facilité, puisque leurs différences flagrantes seraient plus facilement perçues par un apprenant et ainsi rendraient propice la création d'une nouvelle catégorie pour ce son (voir les « $\mathrm{O} »$ dans la figure 1 qui représentent les sons dont les caractéristiques phonologiques diffèrent grandement des catégories de sons de la L1). En effet, selon le SLM une nouvelle catégorie phonologique pour un phonème L2 peut être établie si l'apprenant perçoit des différences phonétiques entre ce son et le son L1 le plus près et plus grandes sont les différences entre ces deux sons, plus la possibilité de détecter les différences entre ceux-ci augmenterait. Toutefois, les phonèmes du système phonologique de la L2 qui n'existent pas dans le système phonologique de la L1, mais qui ressemblent fortement à certains phonèmes de la L1 seront difficiles à apprendre, puisque cette différence parfois mince n'est pas aisément perçue par les apprenants (voir les «+» dans la figure 1 qui représentent des phonèmes dont les caractéristiques diffèrent très peu de certains phonèmes L1). Ce dernier type de phonèmes risque d'être catégorisé à tort comme un phonème similaire du système de la L1 d'un apprenant, car une seule catégorie phonétique pourrait être utilisée pour traiter le son L2 et le son de la L1 qui en est proche. Ces deux sons liés en perception pourraient alors être produits de manière similaire puisqu'ils correspondraient aux mêmes caractéristiques représentées dans une seule catégorie phonologique (Flege, 1995 ; Gut, 2009).

D'autres modèles se sont également intéressés à la perception des phonèmes dans l'apprentissage d'une L2, dont le Modèle d'assimilation perceptuelle (Perceptual Assimilation Model [PAM]) (Best, 1995 ; Best et Tyler, 2007). Bien que celui-ci converge avec le SLM sur plusieurs points, quelques différences sont mises de l'avant par Best et Tyler (2007), notamment le fait que le PAM accorde également une place aux composantes phonétiques et au système phonologique de l'interlangue (et non seulement au contraste entre les phonèmes L2 et ceux de la L1 qui en sont près). Ainsi, selon le PAM, les capacités d'un apprenant à bien distinguer des phonèmes relèvent également de différences au niveau phonétique et articulatoire, plutôt qu'uniquement au niveau phonologique. Par ailleurs, un 
apprenant pourrait assimiler deux phonèmes à une même catégorie phonologique, mais distinguer ceux-ci phonétiquement, c'est-à-dire que leur production ne serait pas pour autant identique (Best, 1995 ; Best et Tyler, 2007). De ce fait, il pourrait alors être possible que pendant l'apprentissage de la L2, les deux catégories phonétiques de ces phonèmes qui, dans l'interlangue de l'apprenant était représentés dans une seule catégorie phonologique, apportent un changement de perception et donc la création d'une nouvelle catégorie phonologique. En somme, une précision notoire de ce modèle réside dans le postulat qu'un apprenant pourrait percevoir la fonction d'un phonème L2 comme étant la même qu'un phonème de sa L1 (et le catégoriser ainsi) tout en percevant une distinction phonétique (articulatoire) entre eux (Best, 1995 ; Best et Tyler, 2007).

Aussi, en plus de considérer les difficultés liées aux systèmes phonologiques en jeu, il faut également se pencher sur la manière d'apprendre des adultes, qui n'est pas la même que celle des enfants (DeKeyser, 2003). DeKeyser (2003) a argumenté que, contrairement aux enfants qui tireraient avantage d'un enseignement qui reflète un milieu naturel, les adultes auraient besoin d'un enseignement formel des règles de la prononciation qui leur permettrait de porter attention aux phonèmes de la L2 pour bien les percevoir et les produire. Plusieurs études (p.ex., Norris et Ortega, 2000 ; DeKeyser, 2003 ; Ellis, Loewen et Erlam, 2006 ; Spada et Tomita, 2010) ont notamment fait ressortir des effets positifs sur la performance des apprenants de L2 de l'enseignement explicite, soit un enseignement pendant lequel il est possible qu'une règle ou une piste vers une règle soit donnée aux apprenants (Norris et Ortega, 2000 ; DeKeyser, 2003 ; Spada et Tomita, 2010), un contraste entre des aspects de la L1 et de la L2 puisse être expliqué (Spada et Tomita, 2010), où l'enseignant pourrait soutenir l'apprentissage à l'aide de la rétroaction de type métalinguistique (Spada et Tomita, 2010) dans un processus qui permet à l'apprenant d'être conscient de l'aspect de la langue visé par l'enseignement (Ellis et coll., 2006). Un exemple d'étude ayant présenté un tel type d'enseignement est celle d'Aliaga-Garcia et Mora (2009). Cette étude a été menée auprès de 36 participants, dont 29 hispanophones qui apprenaient l'anglais à l'université, et 7 locuteurs natifs de l'anglais britannique (modèle de comparaison). Elle visait à mesurer les effets d'un enseignement de la justesse de perception et de production de certains phonèmes anglais considérés difficiles pour ce type d'apprenants (les consonnes occlusives $/ \mathrm{p} \mathrm{t} \mathrm{k} \mathrm{b} \mathrm{d} \mathrm{g/} \mathrm{et} \mathrm{la}$ différence dans la durée de certaines voyelles [/i:/-/I/, /æ/-/-/a:/ et /u:/-/ひ/]). Le traitement a duré six semaines, pendant lesquelles les étudiants recevaient deux heures d'enseignement explicite centré sur l'articulation, la perception et la production de certaines consonnes anglaises ainsi que sur la distinction de voyelles. Les auteurs (Aliaga-Garcia et Mora, 2009) ont constaté à travers un test de discrimination (pour la perception) et une analyse de la parole (VOT des consonnes et, fréquence et durée des voyelles pour la production) que les apprenants ayant reçu ce type d'enseignement ont perçu et produit quelques-uns des phonèmes visés de manière plus juste lorsqu'ils les comparaient au groupe de locuteurs natifs (Aliaga-Garcia et Mora, 2009). D’autres études récentes ont également démontré qu'un enseignement de type explicite pouvait aider les apprenants à bien distinguer des phonèmes précis (voir Aoyama, Flege, Guion, Akahane-Yamada et Yamada, 2004 ; Ellis et coll., 2006 ; Morales Pech et Izquierdo, 2011 ; Lee et coll., 2015). Mis à part l'approche explicite mise en œuvre par ces différentes études, une autre caractéristique commune qu'elles présentent touche la durée assez prolongée des traitements expérimentaux proposés. Plus précisément, dans l'étude de Derwing et coll. (1998), un peu plus de dix-huit heures sur une période de onze semaines ont été consacrées au traitement; dans l'étude d'Aliaga-Garcia et Mora (2009), douze heures ont été consacrées au traitement et, dans celle de Saito et Lyster (2012), 
le traitement était d'une heure par cours, deux fois par semaine pendant deux semaines, soit un total de quatre heures. Néanmoins, considérant la place souvent très restreinte qu'occupe l'enseignement de la prononciation en salle de classe (Foote et coll., 2011) et la difficulté que peuvent éprouver les enseignants à modifier leur plan de cours ou les curriculums en place, l'insertion dans les cours de L2 de séquences d'enseignement d'une telle durée pourrait s'avérer compliquée, voire impossible dans plusieurs cas. Il serait donc pertinent de vérifier dans quelle mesure un enseignement de la prononciation d'une moins longue durée pourrait également avoir un effet sur la performance des apprenants.

Somme toute, les effets d'un enseignement explicite de la perception et de la production de certains phonèmes L2 ont été explorés et les résultats indiquent que ce type d'enseignement améliorerait la performance des apprenants adultes, et ce malgré les défis auxquels ils font face (Aliaga-Garcia et Mora, 2009; Morales Pech et Izquierdo, 2011). C'est d'ailleurs ce que Lee et coll. (2015) ont constaté dans une méta-analyse sur l'efficacité de l'enseignement de la prononciation en L2. Les résultats encourageants corroborent ce qui a été mentionné plus haut : l'enseignement de la perception et de la production des phonèmes à travers un enseignement explicite apporte des gains considérables dans les cours de L2. Cependant, il est intéressant de remarquer que la quasi-totalité des apprenants de ces études était soit des locuteurs ou des apprenants de l'anglais ; en effet, des 86 études faites entre 1982 et 2013 choisies pour cette méta-analyse, 83 de celles-ci avaient l'anglais comme L1 ou langue cible. Dans leur conclusion, Lee et coll. (2015) ont donc recommandé d'élargir le contexte de ce type d'étude en explorant le tout avec d'autres langues que l'anglais, recommandation également formulée par Thomson et Derwing (2015) dans une étude ayant analysé 75 études portant sur l'enseignement de la prononciation. C'est pourquoi, dans le cadre de notre étude, nous nous pencherons sur le cas de francophones adultes apprenant l'allemand L2.

\section{Contexte de l'étude}

Ainsi, les locuteurs francophones adultes qui désirent apprendre l'allemand peuvent se heurter aux difficultés illustrées dans ce qui précède, les langues allemande et française comprenant des systèmes phonologiques différents (Kemp, 2011). Par exemple, dans les deux systèmes, on retrouve le phonème fricatif $/ \mathrm{J} /$ qu'on produit au même lieu d'articulation ([S]). Cependant, en langue allemande, il existe également le phonème fricatif/ç/ qui n'existe pas en français et qui est produit de deux manières différentes (les phones [ç] et [x]) ainsi qu'à un autre lieu d'articulation que le $/ \mathrm{S} /$. Il est primordial pour les apprenants de l'allemand de savoir bien distinguer le phonème $/ \mathrm{g} / \mathrm{du} / \mathrm{ç} /$ (produit [c] ou [x]) comme le montrent les paires minimales suivantes : Kirsche ['krrfa] (cerise) et Kirche ['kırçə] (église), Maschen ['maJən] (mailles) et machen ['maxən] (faire). Effectivement, en allemand, le sens de certains mots n'est accessible que par la bonne production des phonèmes $/ \mathrm{J} /([\mathrm{J}])$ ou /ç/ ([ç] ou $[\mathrm{x}])$. Néanmoins, bien que le phonème /ç/ lorsque produit $[\mathrm{x}]$ (consonne fricative, vélaire, non voisée) soit plus facile à distinguer du phonème / $/$ / (consonne fricative, postalvéolaire, non voisée), car sa production en est plus éloignée, le phonème /ç/ lorsque produit [ç] (consonne fricative, palatale, non voisée) est presque indiscernable pour les apprenants n'ayant pas l'allemand comme langue maternelle, car, bien que différent du phonème $/ \mathrm{J} /$, sa production est presque similaire (Fox, 1990 ; Schiemann et Bölck, 2008). 

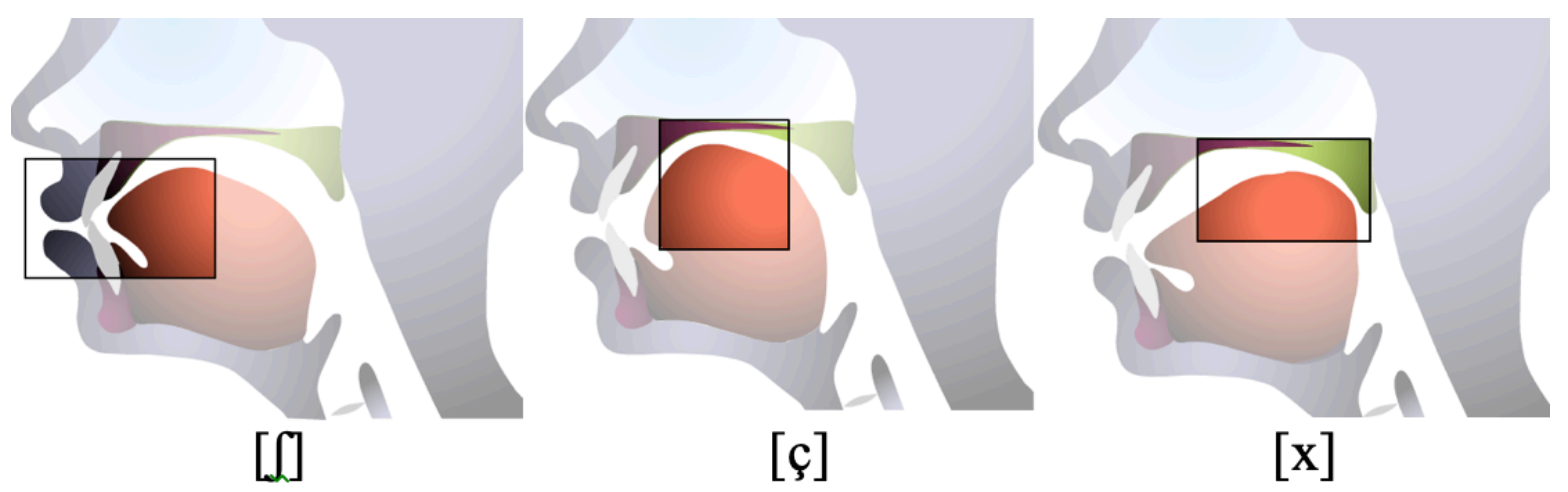

Figure 2. Lieux de production des phones [J], [ç] et [x]. ${ }^{2}$

Il est donc important pour les locuteurs francophones apprenant l'allemand de bien apprendre à percevoir, catégoriser et reproduire ces différents sons pour bien les discriminer et éviter des erreurs qui pourraient nuire à leur intelligibilité, telles qu'affirmer cueillir des églises ['kırçə] ou aller à la cerise ['kırjə] les dimanches.

\section{Objectif de l'étude}

À la lumière de ces informations, cette étude a donc pour objectif de vérifier si, à travers un enseignement explicite sur une courte durée du niveau segmental de la prononciation, donc centré sur des phonèmes, il est possible d'améliorer la prononciation (perception et production) en allemand de locuteurs francophones adultes.

\section{Participants}

\section{Méthodologie}

Cette étude a été menée auprès de 64 participants inscrits dans les trois groupes-cours d'allemand de niveau débutant d'une université montréalaise. Ces apprenants, âgés en moyenne de 24,6 ans (ET : 6,7), étaient des étudiants universitaires inscrits dans un cours d'allemand du premier cycle. La majorité d'entre eux (92,1\% des participants des groupes expérimentaux et $80 \%$ du groupe témoin) ont déclaré avoir le français comme langue maternelle (toutes variations du français confondues) et ont évalué leurs connaissances de l'anglais à un niveau intermédiaire (38,7\%) ou avancé (61,3\%). Deux groupes intacts ont formé les groupes expérimentaux ${ }^{3}(\mathrm{E} 1, n=23 ; \mathrm{E} 2, n=25)$ alors que l'autre groupe a constitué le groupe témoin ( $\mathrm{T}, n=16)$. Outre le nombre de participants, la seule différence marquante constatée entre nos trois groupes est le genre des participants. Effectivement, alors que le groupe E2 comptait presque autant d'hommes que de femmes, le groupe E1 était majoritairement composé de femmes. À l'opposé du groupe E1, le groupe témoin (T) comptait une majorité d'hommes. Enfin, de nos 64 participants, les 48 participants des groupes expérimentaux ont reçu le traitement qui sera détaillé dans la prochaine section.

\section{Phonèmes visés}

Nous avons créé le traitement à partir du livre de phonétique allemande hören sprechen - richtig schreiben : Übungsprogramm zu Phonetik und Rechtschreibung für den 
Unterricht Deutsch als Fremdsprache (Schiemann et Bölck, 2008). En orientant nos choix de phonèmes à partir des modèles SLM (Flege, 1995) et PAM (Best, 1995 ; Best et Tyler, 2007), nous avons identifié cinq sons consonantiques allemands qui ne sont pas dans le système phonologique français, soit les phonèmes $/$ ç/, / $/ \mathrm{ts} /$ et $/ \widetilde{\mathrm{pf}} /$, qui partagent plusieurs caractéristiques avec des phonèmes français et donc pourraient être difficiles à percevoir ou à produire pour nos participants (correspondant aux «+ » dans la figure 1) et le phonème $/ \mathrm{h} /$ ainsi que le phone $[\mathrm{x}]$ (allophone du phonème /ç/), dont les caractéristiques diffèrent de manière plus marquée des phonèmes français, leur apprentissage devrait donc être plus facile que celui des phonèmes précédents (correspondant aux « $\mathrm{O} »$ dans la figure 1). Plus précisément, en ce qui concerne les difficultés anticipées à percevoir et à produire le phonème /ç/, il est possible que les participants distinguent les allophones de ce phonème ([ç] et $[\mathrm{x}])$ ou même le phone $[\mathrm{x}]$ du phonème $/ \mathrm{J} /$ avec lequel le /ç/ (produit [ç]) partage plusieurs caractéristiques, sans pour autant créer une nouvelle catégorie de son pour le phonème /ç/. Effectivement, selon Flege (1995), la distinction entre deux phonèmes (X-Y), n'assure pas la distinction entre d'autres phonèmes $(\mathrm{X}-\mathrm{Z})$. Une distinction phonétique pourrait alors exister entre les phones [ç], [x] et [x], [J], sans qu'une distinction phonologique existe entre les phonèmes $/$ ç/ et $/ \mathrm{f} /$. Aussi, les phonèmes $/ \widetilde{\mathrm{ts}} /$ et $/ \widetilde{\mathrm{pf}} /$ pourraient être difficilement perçus, car ils partagent plusieurs caractéristiques avec les phonèmes /s/ et /f/. Néanmoins, dans la variante du français laurentien (variante parlée où la collecte a eu lieu), on retrouve le phone [ $[$ ts], un allophone du phonème / $\mathrm{t}$ / (Côté, 2005). Cela pourrait faciliter l'apprentissage du phonème /ts/ pour les participants dont la L1 comprend l'allophone [ [ts]. Finalement, plusieurs caractéristiques différencient le phonème $/ \mathrm{h} /$ des phonèmes du français. De plus, les participants ont des connaissances en anglais, langue qui comprend le $/ \mathrm{h} / \mathrm{dans}$ son système phonologique. Ce phonème devrait donc être facilement perçu et produit par nos participants.

\section{Traitement}

Premièrement, à partir du constat selon lequel peu de temps est accordé à l'enseignement de la prononciation dans les cours de L2 (Foote et coll., 2011), il a été établi que notre traitement devait pouvoir être réalisé dans un temps limité, et ce afin de rendre possible l'intégration de l'activité proposée dans les cours de L2 sans nécessairement en altérer le plan préalablement établi par l'enseignant ou le curriculum. Ainsi, notre traitement correspond à une courte série de cinq activités d'une durée de dix à quinze minutes par cours, une fois par semaine, étalé sur cinq semaines.

Le traitement expérimental a donc commencé vers la moitié d'une session universitaire de quinze semaines. Après le prétest qui a eu lieu au cours 9, nous avons expliqué aux participants des groupes expérimentaux le modèle d'apprentissage de la production orale de Flege (1995) (SLM) afin de leur donner la possibilité de comprendre les différences possibles entre les systèmes phonologiques des langues. Ensuite, comme les phonèmes à l'étude étaient des consonnes, nous avons expliqué aux participants quelles étaient les caractéristiques d'une consonne (le mode, le lieu d'articulation et le voisement).

Nous avons poursuivi avec une activité de 10 minutes présentant le phonème /ç/ dès le cours 9. Par la suite, à chaque cours 10 minutes étaient allouées à la présentation d'un phonème différent : le $[\mathrm{x}]$ (allophone du phonème /ç/) (cours 10), suivi du /h/ (cours 11), du $\widetilde{\mathrm{ts}} /($ cours 12$)$ et $\mathrm{du} / \widetilde{\mathrm{pf}} /$ (cours 13). Nous avons également fait un résumé de dix minutes des cinq sons visés au cours 14. 


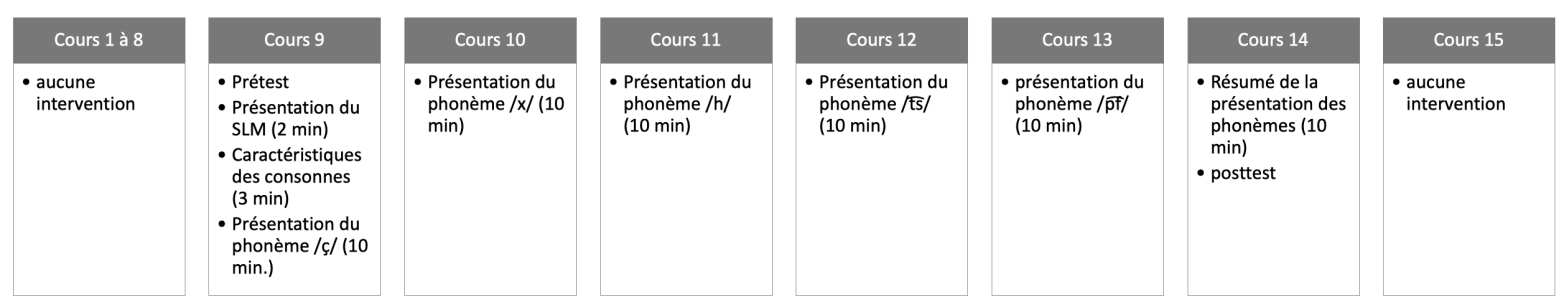

Figure 3. Le déroulement du traitement.

Pour chaque séquence d'activité, nous commencions par présenter une image d'une coupe sagittale qui représentait la production du phonème visé. Grâce à cette image, nous pouvions mettre en valeur le mode et le lieu d'articulation. Ensuite, nous expliquions aux participants comment trouver le lieu d'articulation du phonème à l'étude et leur faisions pratiquer la production de celui-ci à voix haute. Lorsque le son semblait produit adéquatement par le groupe (donc selon les caractéristiques de ces phonèmes dans le livre de phonétique de Schiemann et Bölck [2008]), nous passions à l'étape suivante : la présentation des formes écrites du phonème afin qu'ils puissent le reconnaitre dans les textes. Cette partie était suivie d'une description de l'environnement linguistique du phonème, c'est-à-dire sa position dans une syllabe et les phonèmes qui se retrouvent avant ou après celui-ci. Évidemment, nous donnions constamment des exemples de mots contenant le phonème et, afin de travailler la perception des participants, ceux-ci devaient tout d'abord les écouter plusieurs fois (produits par l'enseignante et ensuite produits par leur collègue en dyade) pour après les répéter, et une activité de discrimination (type $\mathrm{AX}$, écoute de deux mots identiques ou différents) leur était ensuite présentée. De plus, durant toute l'activité, chaque phonème visé était associé à une couleur afin de fournir un signal aux participants, car il était important de les aider à porter attention aux sons, pour qu'ils puissent bien les comprendre et ainsi mieux les percevoir et les reproduire (Schmidt, 2010). Nous terminions la présentation par un résumé de ce qui venait d'être expliqué. Après quoi, nous leur présentions une phrase de type virelangue qui mettait l'accent sur le phonème à l'étude. Ils devaient en premier lieu l'écouter les yeux fermés pour tenter de percevoir les phonèmes visés et ensuite, ils pouvaient lire la phrase en portant attention aux phonèmes. Finalement, lors des cours, l'enseignante responsable du cours, qui était la même pour les deux groupes expérimentaux, s'assurait de faire de la rétroaction de type métalinguistique portant sur l'enseignement explicite décrit cihaut. Par exemple, lorsqu'un texte était projeté au tableau blanc, elle soulignait les sons visés avec les différentes couleurs liées aux phonèmes pour que les participants puissent y porter encore une fois une attention particulière. De plus, lorsque le phonème était mal prononcé par un étudiant, l'enseignante rappelait le lieu et le mode de production du phonème, invitait le participant à écouter les yeux fermés sa prononciation ainsi que la prononciation du participant et à répéter par la suite le phonème. Lorsqu'il était bien prononcé, elle encourageait l'étudiant en lui disant qu'il avait bien positionné sa langue au lieu désiré. Pendant ce temps, le groupe témoin a reçu l'enseignement régulier normalement donné pour ce cours.

Finalement, afin de vérifier la perception et la production des cinq sons consonantiques visés dans cette étude chez nos participants, nous avons collecté des données à l'aide des instruments qui seront présentés dans la prochaine section. 


\section{Instruments}

La performance des participants pour la prononciation des phonèmes a été mesurée à l'aide de deux tâches, soit une tâche de perception et une tâche de production. Deux versions des deux tâches ont été préparées, et ce afin de contrôler l'effet du test sur les résultats obtenus. Premièrement, à l'instar de l'étude d'Aliaga-Garcia et Mora (2009), nos participants ont fait un test de discrimination de type $\mathrm{AX}(/ \mathrm{ç} /-/ \mathrm{J} /, / \mathrm{x} /-/ \mathrm{J} /, / \mathrm{ts} /-/ \mathrm{s} /, / \mathrm{pf} /-/ \mathrm{f} /$ et $/ \mathrm{h} /$-absence de $/ \mathrm{h} /$ ) à l'aide de paires minimales qui contenaient les sons consonantiques allemands de notre étude (quatre paires pour un phonème), ainsi que dix distracteurs que nous avons séparés en nombre égal afin de créer deux versions de difficulté équivalente (voir les annexes A et B). Pour effectuer la tâche, les participants entendaient un enregistrement qui contenait les paires minimales ainsi que les distracteurs et devaient déterminer s'ils percevaient deux mots identiques ou deux mots différents en choisissant une des deux options sur l'ordinateur. Au prétest ainsi qu'au posttest, les participants faisaient deux écoutes consécutives. Lors de la première écoute, les mots étaient prononcés les uns après les autres dans un court délai par une locutrice germanophone et, lors de la deuxième écoute, les mots étaient prononcés de la même manière par un locuteur germanophone, afin que le type de voix n'influence pas les résultats.

La deuxième tâche, inspirée de l'étude de Morales Pech et Izquierdo (2011), servait à évaluer la production des participants. Celle-ci a permis aux apprenants de porter leur attention sur leur production et non sur le sens des mots. En effet, la tâche Word List est une liste de mots contenant trois mots par phonème ciblé ainsi que trois distracteurs et six formules mathématiques qui détournent l'attention des participants des phonèmes cibles et leur donne une pause cognitive (Morales Pech et Izquierdo, 2011). Nous l'avons adaptée à notre recherche en changeant les mots anglais pour des mots allemands qui permettaient de rencontrer les phonèmes visés dans des contextes différents. Entre les deux versions (prétest et posttest), nous avons changé les formules mathématiques et l'ordre d'apparition des mots (voir les annexes $\mathrm{C}$ et $\mathrm{D}$ ). Les participants des trois groupes ont fait les tâches de perception et de production dans un laboratoire informatique au neuvième cours pour le prétest et encore une fois au quatorzième cours pour le posttest.

\section{Dépouillement}

Dans cette étude, nous avons utilisé une approche quantitative de traitement des données recueillies sur la performance en prononciation des participants. Pour la première tâche, il suffisait pour chacun des tests de constater si le participant avait entendu la différence entre les paires minimales en attribuant un 1 aux participants pour une bonne réponse et un 0 pour une mauvaise réponse. Nous avons alors calculé le résultat total pour chaque phonème à chaque moment de la collecte. Également, nous avons fait la somme des totaux pour obtenir un résultat global de la performance de perception pour le prétest et le posttest. Pour la tâche de production, afin qu'un phonème soit considéré comme étant bien produit, la production devait être faite selon la description donnée dans le livre de phonétique de Schiemann et Bölck (2008). Une des chercheures a écouté la totalité des tests des participants afin d'évaluer les phonèmes. Également, afin de nous assurer de la justesse de l'évaluation des phonèmes, nous avons fait appel à une enseignante germanophone pour obtenir un contre-codage de $22 \%$ des résultats pour lesquels les deux correctrices se sont entendues à $94 \%$. Une fois l'évaluation terminée, nous avons regroupé les réponses obtenues 
par phonèmes, ce qui nous a permis d'obtenir un total pour chacun des phonèmes.

Finalement, nous avons pu calculer le total de phonèmes bien produits pour chacun des groupes au prétest et au posttest.

\section{Résultats}

Nous présenterons maintenant les résultats obtenus à la suite des analyses descriptives pour les tâches de perception et de production, et ce, pour les deux temps, c'est-à-dire au prétest et au posttest. Également, afin de vérifier si les différences observées entre les deux temps sont significatives pour ces deux mesures, nous avons effectué des analyses inférentielles. Premièrement, nous avons testé l'effet global grâce à l'ANOVA à mesures répétées. Puis, pour chaque phonème entre les deux temps, nous avons effectué un test de rangs signés de Wilcoxon. Enfin, le test de Kruskal-Wallis nous a permis de vérifier si la différence entre les groupes par phonème aux deux temps (prétest, posttest) était significative.

\section{La tâche de perception}

Les résultats obtenus par les deux groupes aux tests de perception, tant par phonèmes que globalement, sont présentés dans le Tableau 1. Il est à noter que, comme nous avons rencontré des difficultés techniques concernant l'utilisation des ordinateurs pour recueillir les données, le nombre de participants pour chacun des tests n'est donc pas le même.

Tableau 1

Résultats des analyses descriptives pour le test de perception ${ }^{4}$

\begin{tabular}{c|cc|cc|cc}
\hline \multicolumn{7}{c|}{ test de perception } \\
\hline \hline & \multicolumn{2}{|c|}{ Expérimental 1 } & \multicolumn{2}{|c}{ Expérimental 2 } & \multicolumn{2}{c}{ Témoin 1 } \\
\cline { 2 - 7 } & $\begin{array}{c}\text { prétest } \\
(n=23)\end{array}$ & $\begin{array}{c}\text { posttest } \\
(n=18)\end{array}$ & $\begin{array}{c}\text { prétest } \\
(n=25)\end{array}$ & $\begin{array}{c}\text { posttest } \\
(n=20)\end{array}$ & $\begin{array}{c}\text { prétest } \\
(n=16)\end{array}$ & $\begin{array}{c}\text { posttest } \\
(n=14)\end{array}$ \\
\cline { 2 - 8 }$/$ ç/ & $89,1(21,1)$ & $91,7(25,7)$ & $90(20,4)$ & $97,5(11,2)$ & $90,6(20,2)$ & $75(38)$ \\
/x/ & $100(0)$ & $100(0)$ & $100(0)$ & $100(0)$ & $100(0)$ & $96,4(13,4)$ \\
/h/ & $89,1(25,9)$ & $75(30,9)$ & $94(16,6)$ & $92,5(18,3)$ & $100(0)$ & $89,2(28,6)$ \\
/ts/ & $100(0)$ & $100(0)$ & $98(10)$ & $100(0)$ & $93,8(17,1)$ & $96,4(13,7)$ \\
/pf/ & $28,3(33,1)$ & $58,3(42,9)$ & $34(34,5)$ & $60(44,7)$ & $31,3(35,9)$ & $17,9(31,7)$ \\
total & $81,3(8,7)$ & $85(14,7)$ & $83,2(9,9)$ & $90(10,8)$ & $83,1(8,7)$ & $75(10,9)$ \\
\hline \hline
\end{tabular}

Note. Les résultats de ce tableau sont exprimés en pourcentage et représentent le score obtenu (taux de réussite) pour la perception de deux paires minimales par phonème ( 2 items). Pour le total, il y avait donc 10 items évalués.

Perception : résultats globaux. La performance globale des trois groupes aux deux temps de la collecte pour la tâche de perception est illustrée dans la Figure 4. 


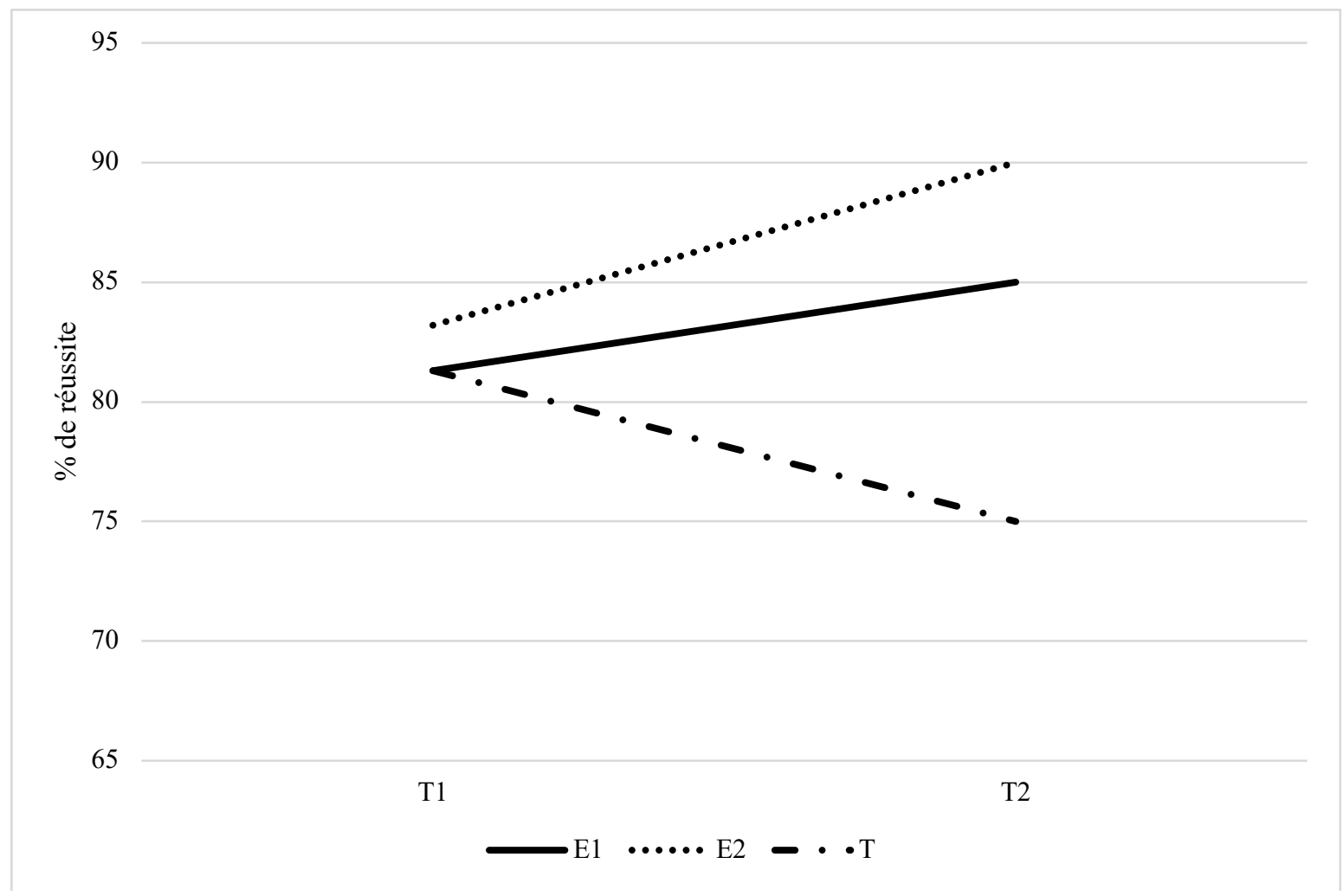

Figure 4. Perception - résultats globaux.

Une analyse de la variance à mesures répétées n'indique aucun effet global du temps, mais un effet global pour le groupe $(\mathrm{F}(2,49)=3,236, \mathrm{p}=.048)$, ainsi qu'une interaction significative temps*groupe $(\mathrm{F}(2,49)=6,999, \mathrm{p}=.002)$.

De façon plus précise, les comparaisons appariées indiquent qu'au prétest, les résultats globaux des trois groupes étaient équivalents, alors qu'au posttest, le groupe E2 a significativement mieux performé que le groupe témoin $(\mathrm{p}=.003)$. Quant à la progression des participants entre le pré et le posttest, nous pouvons remarquer que le résultat total des deux groupes expérimentaux s'est amélioré entre le prétest et le posttest, mais les comparaisons appariées indiquent que seule l'amélioration montrée par le groupe E2 est significative $(\mathrm{p}=.004)$. De son côté, la baisse de performance du groupe T s'avère non seulement surprenante, mais également significative $(p=.026)$. Afin de voir plus en détail comment les performances des participants diffèrent selon les phonèmes visés, nous avons effectué une seconde série d'analyses.

Perception : résultats par phonèmes. Nous pouvons remarquer, dans le Tableau 1 cidessus, que pour la perception des cinq phonèmes visés au prétest les trois groupes ont obtenu des résultats assez similaires et, pour tous les phonèmes sauf pour le / $\widetilde{\mathrm{pf}} /$, assez élevés. Un test de Kruskal-Wallis a confirmé que les minces différences observées n'étaient pas significatives (voir Tableau 2, colonne de gauche). 
Tableau 2

Significations asymptotiques pour le test de Kruskal-Wallis d'échantillons indépendants pour la perception des phonèmes

Test de Kruskal-Wallis d'échantillons indépendants

\begin{tabular}{c|cc}
\hline \hline & prétest & posttest \\
\hline /ç/ & 0,074 & $0,042^{*}$ \\
/x/ & 1 & 0.257 \\
/h/ & 0,226 & 0,074 \\
/ts/ & 0,193 & 0,257 \\
/pf/ & 0,839 & $0,011^{*}$ \\
\hline \hline
\end{tabular}

Toutefois, la lecture du Tableau 2 montre que les moyennes des groupes diffèrent davantage au posttest. Les résultats au test de Kruskal-Wallis montrent en effet que les groupes diffèrent significativement au posttest pour les phonèmes /ç/ et / $/$ ff/ (voir Tableau 2 , colonne de droite). Pour ces deux phonèmes, ces différences sont principalement attribuables à la performance du groupe témoin, significativement moins élevée que celle du groupe E1 au posttest pour le phonème /ç/ $(\mathrm{T}-\mathrm{E} 1: \mathrm{p}=.028)$ et que celle des deux groupes expérimentaux $(\mathrm{T}$ $\mathrm{E} 1: \mathrm{p}=.038, \mathrm{~T}-\mathrm{E} 2: \mathrm{p}=.024)$ pour le $/ \widetilde{\mathrm{pf}} /$.

En ce qui concerne la progression, pour chaque phonème des trois groupes entre le prétest et le posttest, un test de classement de Wilcoxon nous permet de remarquer, d'abord, que la performance du groupe témoin est restée stable pour l'ensemble des phonèmes (voir Tableau 3).

Tableau 3

Le test de classement de Wilcoxon pour la perception

\begin{tabular}{|c|c|c|c|c|c|c|c|c|c|c|}
\hline \multicolumn{11}{|c|}{ Test de classement de Wilcoxon (perception) } \\
\hline \multirow[t]{2}{*}{ groupes } & \multicolumn{2}{|c|}{$|c|$} & \multicolumn{2}{|c|}{$|x|$} & \multicolumn{2}{|r|}{$/ \mathrm{h} /$} & \multicolumn{2}{|r|}{$|\mathrm{ts}|$} & \multicolumn{2}{|c|}{ /pf/ } \\
\hline & 2 & sig.asymp. & $z$ & sig.asymp. & 2 & sig.asymp. & 2 & sig.asymp. & 2 & sig.asymp. \\
\hline E1 & $-0,447$ & 0,655 & 0,000 & 1,000 & $-2,236$ & $0,025^{*}$ & 0,000 & 1,000 & $-2,226$ & $0,026^{*}$ \\
\hline E2 & $-1,414$ & 0,157 & 0,000 & 1,000 & 0,010 & 1,000 & 0,000 & 1,000 & $-2,762$ & $0,006^{*}$ \\
\hline$T$ & $-1,633$ & 0,102 & $-1,000$ & 0,317 & $-1,342$ & 0,180 & 1,000 & 0,317 & $-0,816$ & 0,414 \\
\hline
\end{tabular}

Pour les groupes expérimentaux, on observe une différence significative de perception $\mathrm{du}$ prétest et au posttest pour le phonème $/ \widehat{\mathrm{pf}} / \mathrm{chez}$ les groupes $\mathrm{E} 1$ et $\mathrm{E} 2$, qui se sont tous deux 
améliorés entre les deux temps $(\mathrm{E} 1: \mathrm{p}=.026 ; \mathrm{E} 2: \mathrm{p}=.006)$ et pour le phonème $/ \mathrm{h} / \mathrm{pour}$ le groupe E1 qui a eu plus de difficulté à percevoir ce son au posttest $(\mathrm{p}=.025)$.

\section{La tâche de production}

Nous avons rassemblé les résultats du test de production pour chacun des phonèmes ainsi que pour le total des phonèmes dans le Tableau 4. Comme nous avons fait face à d'autres problèmes techniques pour l'enregistrement de la production que ceux survenus lors de la tâche de perception, le nombre de participants pour chaque groupe pour cette tâche n'est pas le même que pour la tâche précédente.

Tableau 4

Résultats des analyses descriptives pour le test de production

\begin{tabular}{c|cc|cc|cc}
\hline \multicolumn{7}{c}{ test de production contrôlée } \\
\hline \hline & \multicolumn{2}{|c|}{ Expérimental 1 } & \multicolumn{2}{c}{ Expérimental 2 } & \multicolumn{2}{c}{ Témoin 1 } \\
\cline { 2 - 8 } & prétest & posttest & prétest & posttest & prétest & posttest \\
& $(n=16)$ & $(n=15)$ & $(n=21)$ & $(n=20)$ & $(n=15)$ & $(n=13)$ \\
\cline { 2 - 8 } /ç/ & $27,1(25)$ & $71,1(33)$ & $38,1(30,3)$ & $63,3(35,7)$ & $31,1(23,5)$ & $35,9(31,8)$ \\
/x/ & $29,2(31,9)$ & $44,4(34,9)$ & $30,2(40,7)$ & $48,3(38,2)$ & $51,1(39,6)$ & $51,3(37,6)$ \\
/h/ & $72,9(30,4)$ & $97,8(8,6)$ & $76,2(33,6)$ & $100(0)$ & $100(0)$ & $94,9(12,5)$ \\
/ts/ & $27,1(38,9)$ & $88,9(20,6)$ & $57,1(38,2)$ & $76,7(30,8)$ & $44,4(39,2)$ & $51,3(35)$ \\
/pf/ & $87,5(24)$ & $100(0)$ & $90,5(18,7)$ & $100(0)$ & $86,7(24,6)$ & $92,3(14,6)$ \\
total & $48,8(17,2)$ & $80,44(13,7)$ & $58,4(17,4)$ & $77,7(16,7)$ & $62,7(14)$ & $65,1(14,4)$ \\
\hline \hline
\end{tabular}

Note. Les résultats de ce tableau sont exprimés en pourcentage et représentent le score obtenu pour la production de chaque phonème dans 3 mots différents ( 3 items par phonème). Pour le total, il y avait donc 15 items évalués.

Production : résultats globaux. La performance globale des trois groupes aux deux temps de la collecte pour la tâche de production est illustrée dans la Figure 5. 


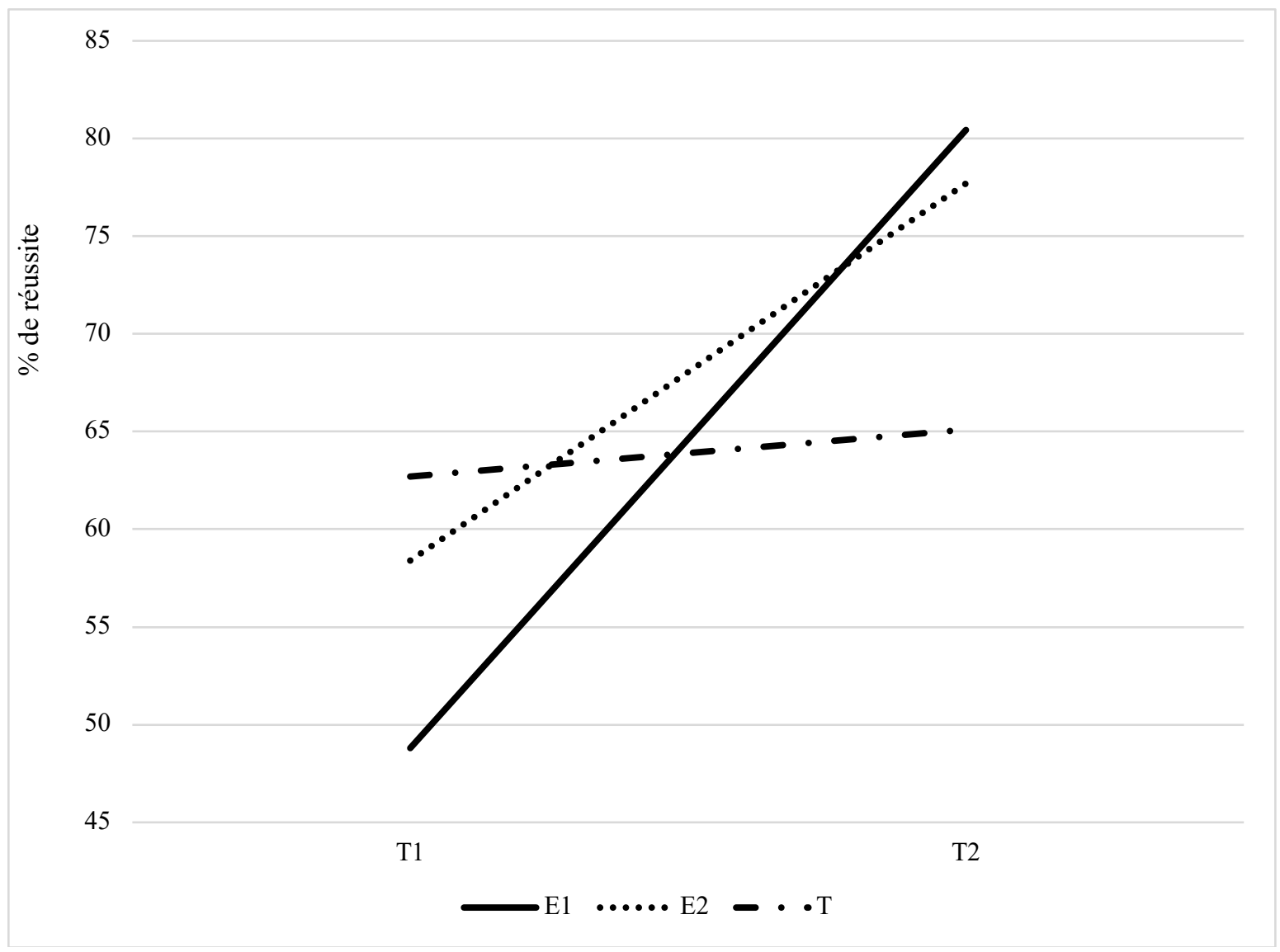

Figure 5. Production - résultats globaux.

Contrairement à ce qui avait été observé pour la perception totale, l'analyse de la variance à mesures répétées indique un effet global du temps $(\mathrm{F}(1,38)=48,973, \mathrm{p}<.001)$, mais aucun effet global associé au groupe. Une interaction significative temps*groupe (F $(2,38)=7,996, p=.001)$ est également observée.

L'analyse détaillée offerte par les comparaisons appariées indique que les trois groupes ont obtenu des résultats statistiquement comparables au prétest ainsi qu'au posttest, mais que les groupes E1 et E2 se sont améliorés de façon significative entre le prétest et le posttest $(E 1: p<.001 ; E 2: p<.001)$, alors que la performance du groupe témoin est restée stable. Nous verrons, dans ce qui suit, comment ces résultats s'articulent selon les phonèmes visés.

Production : résultats par phonèmes. La production des cinq phonèmes au prétest semble assez similaire dans les trois groupes, ce qu'un test de Kruskal-Wallis a confirmé pour tous les phonèmes à l'exception du $/ \mathrm{h} /$ (voir Tableau 5 , colonne de gauche). Pour ce phonème, le groupe témoin a significativement mieux performé que le groupe $\mathrm{E} 1(\mathrm{p}=.028)$ et que le groupe E2 $(\mathrm{p}=.011)$. 
Tableau 5

Significations asymptotiques pour le test de Kruskal-Wallis d'échantillons indépendants pour la production

Test de Kruskal-Wallis d'échantillons indépendants

\begin{tabular}{c|cc}
\hline \hline & prétest & posttest \\
\hline /ç/ & 0,555 & $0,025^{*}$ \\
/x/ & 0,186 & 0,886 \\
/h/ & $0,007^{*}$ & 0,210 \\
/ts/ & 0,065 & $0,006^{*}$ \\
/pf/ & 0,948 & $0,015^{*}$ \\
\hline \hline
\end{tabular}

$*$ sig $<0,05$

Comme pour le test de perception, les moyennes des groupes diffèrent davantage au posttest. Les résultats au test de Kruskal-Wallis montrent en effet que les groupes diffèrent significativement au posttest pour les phonèmes /ç/, / $/ \mathrm{ts} /$ et $/ \widehat{\mathrm{pf}} /$ (voir Tableau 5 , colonne de droite). Pour le /ç/ et le /ts/, la différence se situe entre le groupe T et le groupe E1 (/ç/, $\mathrm{p}=.028 ; / \widetilde{\mathrm{ts}} /, \mathrm{p}=.05)$, alors que pour le $/ \widetilde{\mathrm{pf}} /$, ce sont les deux groupes expérimentaux qui diffèrent du groupe témoin $(\mathrm{T}-\mathrm{E} 1: \mathrm{p}=.038, \mathrm{~T}-\mathrm{E} 2: \mathrm{p}=.024)$.

La progression des trois groupes entre le prétest et le posttest a ensuite été analysée à l'aide d'un test de classement de Wilcoxon (voir Tableau 6).

Tableau 6

Test de classement de Wilcoxon pour la production

\begin{tabular}{|c|c|c|c|c|c|c|c|c|c|c|}
\hline \multicolumn{11}{|c|}{ Test de classement de de Wilcoxon } \\
\hline \multirow[b]{2}{*}{ groupes } & \multicolumn{2}{|c|}{$\mid c /$} & \multicolumn{2}{|r|}{$|x|$} & \multicolumn{2}{|c|}{$/ \mathrm{h} /$} & \multicolumn{2}{|c|}{ /ts/ } & \multicolumn{2}{|c|}{ /pf/ } \\
\hline & Z & sig.asymp. & Z & sig.asymp. & Z & sig.asymp. & z & sig.asymp. & Z & sig.asymp. \\
\hline E1 & $-2,810$ & $0,005^{*}$ & $-1,000$ & 0,317 & $-2,070$ & $0,038^{*}$ & $-2,521$ & $0,012^{*}$ & $-1,414$ & 0,157 \\
\hline E2 & $-2,887$ & $0,004^{*}$ & $-2,428$ & $0,015^{*}$ & $-2,414$ & $0,016^{*}$ & $-2,230$ & $0,026^{*}$ & $-2,121$ & $0,034^{*}$ \\
\hline $\mathrm{T}$ & $-0,378$ & 0,705 & $-0,690$ & 0,490 & $-1,414$ & 0,157 & $-0,378$ & 0,705 & $-1,089$ & 0,276 \\
\hline
\end{tabular}

$*_{\text {sig }}<0,05$

Ces analyses montrent une amélioration significative des groupes expérimentaux sur l'ensemble des phonèmes pour le groupe $\mathrm{E} 2$, et sur trois des cinq phonèmes $(/ \mathrm{ç} /, / \mathrm{h} /, / \mathrm{ts} /)$ pour le groupe E1. Le groupe témoin, quant à lui, ne s'est amélioré sur aucun des phonèmes entre les deux temps de la collecte.

En somme, le traitement expérimental semble avoir bien servi les groupes expérimentaux tant pour la perception que la production de ces phonèmes. 


\section{Discussion}

Dans cette étude, nous avons recueilli des données sur la perception et la production de phonèmes ciblés par des apprenants francophones adultes d'un cours d'allemand débutant. Ces participants étaient répartis dans trois groupes différents : le groupe expérimental 1 et le groupe expérimental 2, qui ont reçu un enseignement explicite de la perception et de la production de certains phonèmes allemands, ainsi que le groupe témoin ayant reçu l'enseignement normalement donné pour ce cours.

Notre traitement, basé sur l'enseignement explicite, avait pour but d'offrir l'occasion aux apprenants de porter attention à leur système phonologique pour qu'ils puissent comprendre comment ce système diffère de celui de la L2 comme Derwing et Munro (2005) et Lee et coll. (2015) l'ont suggéré. Les résultats de notre étude démontrent en effet que notre traitement basé sur l'enseignement explicite a aidé les apprenants des groupes expérimentaux à percevoir les différences et les similitudes entre les deux systèmes phonologiques du français et de l'allemand. Effectivement, comme les apprenants adultes, d'une part, sont moins sensibles aux sons différents de leur L1 et que, d'une autre part, ont leur système phonologique L1 complètement développé venant ainsi interférer dans l'apprentissage des sons d'une L2 (Flege et coll., 1999; Iverson, Kuhl, Akahane-Yamada, Diesch, Tohkura, et Kettermann, 2003 ; Baker et coll., 2008), ceux-ci peuvent avoir tiré avantage d'un tel type d'enseignement qui permet de porter attention à des aspects de la langue qui sont plus difficiles à remarquer seuls (DeKeyser, 2003). Par ailleurs, l'enseignement régulier ne semble pas avoir eu le même effet sur le groupe témoin, car les participants de celui-ci ont obtenu des résultats moins concluants que ceux des groupes expérimentaux. En somme, les résultats ont permis de démontrer que les effets d'un enseignement explicite de la perception et de la production de certains phonèmes allemands chez les apprenants francophones adultes sont bénéfiques pour mieux percevoir et produire des phonèmes allemands qui n'existent pas dans leur L1, ce qui corrobore les résultats d'études antérieures (p.ex., Morales Pech et Izquierdo, 2011 ; Aliaga-Garcia et Mora, 2009).

En outre, nous avons observé que la production des deux groupes expérimentaux s'est améliorée significativement entre le prétest et le posttest et que, pour la perception, bien que les deux groupes aient montré des gains, seuls ceux de groupe E2 étaient significatifs. Du côté du groupe témoin qui a reçu l'enseignement régulier, nous avons remarqué qu'il y a eu une diminution significative de la perception des phonèmes visés et aucune amélioration significative pour la production. Best et Taylor (2007) ont expliqué que la possibilité de créer une nouvelle catégorie de son arrivait tôt dans l'apprentissage, car plus celui-ci avançait, plus les apprenants auraient tendance à détourner leur attention des aspects phonologiques vers des structures linguistiques de plus haut niveau. Ainsi, il serait possible que sans un enseignement explicite, l'attention des participants du groupe témoin n'ait pas été suffisamment dirigée vers certains aspects phonologiques et, qu'avec le temps, ils aient concentré leur attention vers d'autres aspects de la langue cible. Par conséquent, ceci pourrait fournir une piste explicative quant à la raison pour laquelle nous n'avons pas constaté d'amélioration significative de leur production et même une diminution significative de leur perception des phonèmes visés. Il est alors possible que certaines caractéristiques de notre traitement (par exemple, la mise en évidence du contraste entre la L1 et la L2, la rétroaction métalinguistique, la pratique de la perception et de la production, et la présentation d'indices contextuels) puissent avoir contribué aux gains observés chez les groupes expérimentaux, 
sans toutefois que nous puissions déterminer l'impact précis de chacune de ces caractéristiques qui auraient pu aider les apprenants à porter attention aux aspects visés, rendant plus propice la création de nouvelles catégories de son.

Également, ce qui est particulièrement intéressant et qui distingue ce traitement d'autres études est le temps de classe très limité que nous avons mis en place pour l'enseignement de la prononciation. En effet, il peut être difficile de suggérer à des enseignants disposant souvent de peu de temps de classe de modifier leurs pratiques ou le curriculum de cours pour laisser à l'enseignement de la prononciation la place que proposaient plusieurs études antérieures. Concrètement, si nous prenons l'exemple d'AliagaGarcia et Mora (2009) et Derwing et coll. (1998), ceux-ci ont consacré environ deux heures de cours par semaine à ce type d'enseignement. De notre côté, nous voulions proposer un enseignement explicite sous forme d'activité d'introduction des phonèmes différents des systèmes de la L1 et de la L2 d'une durée de dix à quinze minutes par semaine.

Conséquemment, puisque le cours universitaire dans lequel nous avons collecté nos données compte 45 heures d'enseignement, notre intervention représentait seulement 2,2\% du temps de classe (environ 1 heure). Considérant que moins de $5 \%$ du temps de classe est normalement alloué à l'enseignement de la prononciation (Foote et coll., 2011), le but était d'altérer le moins possible les plans de cours et d'éviter d'imposer un changement important pour l'enseignant. En effet, l'enseignant n'avait qu'à intégrer le dix à quinze minutes d'activité et à assurer une rétroaction corrective de type métalinguistique durant les cours pour pouvoir obtenir des résultats encourageants, qui rejoignent les résultats obtenus précédemment dans différentes études (p.ex., Aliaga-Garcia et Mora, 2009 ; Morales Pech et Izquierdo, 2011 ; Saito et Lyster, 2012 ; Saito, 2015).

Cela laisserait par ailleurs du temps pour travailler le niveau suprasegmental de la prononciation, également important pour développer l'intelligibilité ou la compréhensibilité des apprenants (Derwing et Munro, 2005 ; Lee et coll., 2015). De cette manière, il serait possible de s'assurer que le temps dédié à la prononciation est correctement orienté. En effet, dans une étude longitudinale basée sur l'observation de corpus en enseignement de l'anglais L2, Foote, Trofimovich, Collins et Urzúa (2016) ont constaté que la plupart des moments de classe réservés à l'enseignement de la prononciation n'étaient pas planifiés. En fait, lorsque les enseignants utilisaient du temps de classe pour enseigner la prononciation, c'était principalement pour corriger les erreurs des apprenants (Foote et coll., 2016). C'est pourquoi, en ciblant les composantes segmentale et suprasegmentale à travers des activités planifiées comme notre traitement, le peu de temps réservé à la prononciation pourrait devenir plus efficient.

Néanmoins, les résultats varient selon les phonèmes ciblés et ces variations ne concordent pas toujours avec nos prédictions orientées par certains modèles de perception. En effet, notre analyse des phonèmes visés prédisait que les phonèmes /ç/ (consonne fricative, palatale), /ts/ (consonne affriquée, alvéolaire) et /pf/ (consonne affriquée, labiodentale) seraient difficiles à apprendre, car ils sont semblables à des consonnes du français $(/ \mathrm{J} /$ [consonne fricative, postalvéolaire], /s/ [consonne fricative, alvéolaire], /f/ [consonne fricative, labiodentale]) alors que les sons $/ \mathrm{h} /$ (consonne fricative, glottale) et [x] (consonne fricative, vélaire), dont la différence avec des phonèmes du français est saillante, auraient dû être plus faciles à apprendre. En effet, un apprenant doit pouvoir remarquer une différence entre deux sons pour pouvoir établir une nouvelle catégorie phonétique et plus les différences entre des sons sont nombreuses, plus grande sont les chances qu'un apprenant puisse les remarquer (Flege, 1995). 
Toutefois, en nous penchant sur les résultats obtenus lors de la tâche de perception, nous observons que le phonème / $\widehat{\mathrm{pf}} /$ était le phonème pour lequel la performance des participants s'est avérée la plus faible à la fois au prétest et au posttest pour tous les groupes. Cependant, ce phonème est aussi celui pour lequel les groupes expérimentaux ont observé la plus grande amélioration. Le phonème / $/ \mathrm{pf} /$, pouvant être considéré difficile, car il partage plusieurs caractéristiques avec le phonème /f/, a alors bénéficié de l'enseignement explicite, à l'instar des résultats obtenus dans l'étude de Saito et Lyster (2012). Cependant, le phonème /h/, qui aurait dû facilement être perçu, puisque ces caractéristiques diffèrent d'autres phonèmes, ne l'a pas été : la performance de perception de tous les groupes a diminué entre le pré et le posttest et cette différence était significative pour le groupe E1. Ce résultat peut être éclairé par certains travaux documentant la difficulté pour des francophones apprenant l'anglais de traiter le phonème /h/ (p.ex., John et Cardoso, 2009 ; Mah, Goad et Steinhauer, 2016). John et Cardoso (2009) ont expliqué que les francophones qui apprennent l'anglais ont tendance à faire l'élision du phonème /h/ lorsqu'il est nécessaire et de faire un $/ \mathrm{h} /$ épenthèse lorsque celui-ci n'est pas requis. Mah et coll. (2016) ont suggéré quant à eux que ce problème réside dans la construction d'une représentation phonologique adéquate pour le /h/ dans l'interlangue des apprenants. Ainsi, il se pourrait que, lors de leur apprentissage de l'anglais, nos participants n'aient pas porté suffisamment d'attention à ce phonème et plus le développement de cette L2 s'est poursuivi, plus leur attention s'est tournée vers d'autres aspects linguistiques (Best et Tyler, 2007). Alors que nous avons cru que le niveau d'anglais de nos participants (intermédiaire [38,7\%] et avancé [61,3\%]) aurait pu faciliter leur perception et leur production du phonème $/ \mathrm{h} /$, car une catégorie phonologique pour ce phonème aurait pu déjà avoir été établie, tel ne semble pas avoir été le cas. Cette particularité des francophones peut expliquer nos résultats et gagnerait à être explorée davantage dans leur apprentissage de l'allemand.

Du côté de la tâche de production, les phonèmes qui ont le plus bénéficié du traitement dans les deux groupes expérimentaux sont les phonèmes /ç/ et / ts /, prédits comme étant difficiles selon notre analyse, car il s'agissait de sons nouveaux. La performance des deux groupes expérimentaux pour la production du phonème $/ \mathrm{h} / \mathrm{a}$ également augmenté de manière significative entre le pré et posttest, et ce, même si ce phonème avait été plutôt difficile à percevoir dans la tâche de perception. Selon le PAM, il est possible que des apprenants développent une catégorie phonétique pour un phonème, sans qu'une catégorie phonologique le soit (Best et Tyler, 2007). Puisque les participants des groupes expérimentaux ont significativement mieux produit le phonème $/ \mathrm{h} /$ après notre traitement, celui-ci pourrait les avoir aidés à développer une catégorie phonétique pour ce phonème. L'enseignement explicite pourrait donc avoir fourni l'occasion aux apprenants des groupes expérimentaux de comprendre et de saisir les différences subtiles entre les phonèmes de leur système phonologique L1 et ceux de la langue cible pour ensuite pouvoir bien les prononcer.

Si le SLM et le PAM ont représenté une piste d'analyse intéressante des phonèmes à l'étude, il serait également pertinent de se pencher sur d'autres facteurs qui définissent les difficultés d'apprentissage des sons. En effet, nos résultats semblent indiquer qu'il y ait d'autres facteurs en cause pour déterminer la difficulté de l'apprentissage d'un phonème que les caractéristiques de celui-ci par rapport à ceux de la L1. Par exemple, les sons /ç/, / $\overparen{\text { ts}} /$ et $/$ pf/ pourraient être plus difficiles à apprendre que les sons $[\mathrm{x}]$ et $/ \mathrm{h} /$, en raison du nombre de caractéristiques semblables à certains phonèmes L1. Cependant, nous avons constaté que les participants n'ont pas semblé avoir de la difficulté à percevoir le son [x], mais ont eu des problèmes quant à sa production. À l'inverse, le phonème / $/ \overline{\mathrm{pf}} / \mathrm{a}$ été difficilement perçu, mais 
relativement bien produit. En somme, les difficultés ou facilités d'apprentissage des phonèmes n'étaient pas les mêmes en perception et en production. Flege (1995) a expliqué que des erreurs en production pouvaient avoir une base perceptuelle, mais que la perception n'était pas la seule explication possible. Également, un apprenant peut développer des catégories phonétiques et donc produire distinctement certains phonèmes, sans nécessairement percevoir ces phonèmes comme faisant partie de deux catégories phonologiques différentes (Best, 1995 ; Best et Tyler, 2007).

De plus, la difficulté qu'ont les francophones à bien percevoir et produire le phonème /h/ en anglais a été relevée dans certaines études (voir John et Cardoso, 2009; Mah et coll., 2016). Ce problème devrait donc être pris en considération lorsqu'une L2 comprenant ce phonème est enseignée à des francophones. Finalement, nous proposons aussi de prendre en considération la fréquence d'un son pour juger de l'importance de son enseignement. En effet, le phonème /pf/ est très peu fréquent en allemand et n'apparaissait pas dans les mots en apprentissage dans le cours où a eu lieu notre intervention. Le caractère marqué de certains phonèmes (plus spécifique, moins fréquent, inattendu, moins saillant) peut rendre leur apprentissage plus complexe (Eckman, 1977). Eckman (1977, p.320) a expliqué qu'un phénomène dans certaines langues (dans le cas de notre étude, une consonne affriquée) pouvait être plus marqué qu'un autre (par exemple une consonne fricative), si sa présence (consonne affriquée) dans une langue sous-entendait la présence de l'autre (consonne fricative), mais que la présence de l'autre (consonne fricative) ne sous-entendait pas sa présence (consonne affriquée). Alors, puisque des consonnes fricatives existent dans certaines langues sans que des consonnes affriquées ne se retrouvent dans leur système phonologique (comme dans le cas du français où le /f/ fait partie du système phonologique sans que le / $/ \overline{\mathrm{pf}} /$ n'y soit), le phonème / $/ \mathrm{pf} /$ serait donc plus marqué que le phonème /f/ et poserait plus de difficulté lors de l'apprentissage, ce qui pourrait expliquer nos résultats. Toutefois, bien que le /ts/ soit également une consonne affriquée, une particularité régionale pourrait, par exemple, avoir joué un rôle dans la performance des participants relativement à ce phonème qui a été mieux perçu que le $/ \widehat{\mathrm{pf}} /$. Plus précisément, une affrication existe dans la variante $\mathrm{du}$ français québécois pour les consonnes dentales / $t /$ et $/ \mathrm{d} /$ lorsqu'elles précèdent une voyelle haute et antérieure (Côté, 2005) et pourrait influencer l'apprentissage du phonème /ts/ allemand chez des apprenants québécois. Ceci met en lumière l'importance d'accorder une place aux similarités phonétiques entre la L1 et la L2 et non seulement aux catégories phonologiques.

En somme, beaucoup de pistes restent à être explorées pour bien déterminer la difficulté d'apprentissage d'un phonème, ce qui par la suite permettrait d'adapter l'enseignement en conséquence. Par exemple dans une étude en 2006, Munro et Derwing se sont intéressés au lien entre la charge fonctionnelle (functional load), c'est-à-dire l'importance d'un contraste entre deux phonèmes selon, entre autres, la fréquence d'apparition dans des paires minimales, leur place dans un mot, etc., et l'intelligibilité. Après avoir fait une étude où 13 anglophones évaluaient la performance de 23 locuteurs du cantonais qui prononçaient des énoncés comprenant des phonèmes ayant une charge fonctionnelle différente, ils ont rapporté que la distinction de certains phonèmes d'une L2 pouvait avoir une influence sur l'intelligibilité des apprenants et que plus la charge fonctionnelle de ces phonèmes était élevée plus l'influence serait importante (Munro et Derwing, 2006). Il est alors important de considérer la charge fonctionnelle des phonèmes d'une L2 pour orienter l'enseignement segmental vers les phonèmes qui aideront l'amélioration de l'intelligibilité (Munro et Derwing, 2006) ou de la compréhensibilité des 
apprenants (Thomson et Derwing, 2015). C'est également ce que Jenkins (2002) avait rapporté dans son étude sur l'intelligibilité entre des locuteurs non natifs adultes apprenant l'anglais.

\section{Les limites}

Malgré ses résultats encourageants, notre étude comporte quelques limites. De toute évidence, nous ne pouvons pas généraliser nos résultats, car notre échantillon n'est pas représentatif de la population. Effectivement, d'un côté, nous n'avons pas obtenu les mêmes résultats pour les deux groupes expérimentaux, le groupe E2 ayant mieux performé que le groupe E1 et, d'un autre côté, nous n'avions qu'un seul groupe témoin pour vérifier les effets de notre traitement.

Finalement, nous relevons une limite sur un de nos instruments de mesure et sur les moments de collecte de données. La tâche de production comportait au prétest et au posttest les mêmes mots cibles, un effet du test pourrait avoir influencé les résultats pour les trois groupes. Aussi, cette tâche consistait à lire une liste de mots comprenant les phonèmes cibles. Toutefois, le phonème /ç/ est produit par les allophones [ç] et [x]. Les deux sons sont alors représentés par les lettres «ch » à l'écrit. Il est donc possible que cette caractéristique ait pu influencer nos résultats puisque certains participants pourraient avoir des difficultés à comprendre le contexte dans lequel le [ç] ou le [x] devrait être produit, et ce, malgré les activités dans lesquelles l'environnement des phones était présenté. Il faudrait alors vérifier si une activité de plus ( $6^{\mathrm{e}}$ activité de 10 minutes) pourrait aider les apprenants à bien distinguer les différents contextes dans lesquels ces allophones apparaissent. De plus, la collecte de données a été faite durant les mêmes cours, mais à des moments différents durant les cours, car les moments d'inclusion de ceux-ci étaient à la discrétion des enseignants, cela pourrait avoir influencé la qualité des réponses des apprenants.

\section{Les pistes de recherche}

Somme toute, des implications pédagogiques intéressantes ressortent de cette étude dans laquelle nous avons présenté un traitement qui se différencie des autres études d'une part par le choix des langues source et cible de nos participants et d'une autre part, par la durée de celui-ci. Également, sans négliger les difficultés que peuvent avoir les enseignants à modifier leur plan de cours, cette étude offre une activité qui pourrait venir s'inscrire dans le programme d'un cours de langue sans trop en altérer le contenu et qui, selon nos résultats, pourrait aider les apprenants avec la perception et la production des phonèmes de la langue cible qui n'existent pas dans leur L1. Toutefois, comme notre traitement se penchait uniquement sur le niveau segmental de la prononciation, il serait intéressant de trouver une manière de l'adapter au niveau suprasegmental afin de s'assurer que les apprenants améliorent de différentes façons leur intelligibilité et leur compréhensibilité (Levis, 2005 ; Derwing et Munro, 2005 ; Lee et coll., 2015; Thomson et Derwing, 2015).

Finalement, puisque nous avons fait face à une contrainte de temps, nous n'avons pas eu la chance de collecter des données lors d'un posttest différé. Il serait alors intéressant que les études futures s'assurent d'obtenir des résultats quelque temps après le traitement pour ainsi enrichir les connaissances actuelles sur les effets à long terme de ce type d'enseignement (Lee et coll., 2015 ; Thomson et Derwing, 2015). De plus, selon Thomson et 
Derwing (2015), l'enseignement explicite pourrait permettre aux apprenants d'améliorer leur prononciation en L2 après le traitement si celui-ci leur a fourni la possibilité de prendre conscience de certaines caractéristiques de la prononciation en L2. Un posttest différé permettrait donc de retirer différentes informations pertinentes pour ce domaine d'étude. Plus de recherches sont ainsi nécessaires pour confirmer l'étendue des effets de l'enseignement explicite dans l'enseignement de la prononciation.

La correspondance devrait être adressée à Isabelle Wouters.

Courriel : wouters.isabelle@uqam.ca

\section{Notes}

${ }^{1}$ Dans cette étude, L2 fait référence à toutes langues apprises après la langue maternelle.

${ }^{2}$ Les coupes sagittales proviennent du site Sounds of Speech (University of Iowa, 2014).

${ }^{3}$ Puisque les participants recevant le traitement faisaient partie de deux groupes-cours différents, nous avons préféré analyser leurs résultats séparément et, ainsi, avons deux groupes expérimentaux distincts.

${ }^{4}$ Contraste pour chaque phonème : /ç/-/ $/, / \mathrm{x} /-/ \mathrm{S} /, \widehat{\mathrm{ts}} /-/ \mathrm{s} /, / \mathrm{pf} /-/ \mathrm{f} /$ et $/ \mathrm{h} /$-absence de $/ \mathrm{h} /$

\section{Références}

Aliaga-Garcia, C., et Mora, J. (2009). Assessing the effects of phonetic training on L2 sound perception and production. Recent Research in Second Language phonetics/phonology: Perception and production, 2-31.

Aoyama, K., Flege, J. E., Guion, S. G., Akahane-Yamada, R., et Yamada, T. (2004). Perceived phonetic dissimilarity and L2 speech learning: The case of Japanese $/ \mathrm{r} /$ and English /1/ and /r/. Journal of Phonetics, 32(2), 233-250. doi :10.1016/S00954470(03)00036-6

Baker, W., Trofimovich, P., Flege, J. E., Mack, M., et Halter, R. (2008). Child-adult differences in second language phonological learning: The role of cross-language similarity. Language and Speech, 51(4), 317-342.

Best, C. T. (1995). A direct realist view of cross-language speech perception. Dans W. Strange (Ed.), Speech Perception and Linguistic Experience: Issues in Cross Language Research. (pp. 171-204). Timonium, MD: York Press.

Best, C.T. et Tyler, M.D. (2007). Nonnative and second-language speech perception: Commonalities and complementaries. Dans M.J. Munro et O.-S. Bohn (Eds.), Second language speech learning: The role of language experience in speech perception and production (pp. 13-34). Amsterdam: John Benjamins.

Cardoso, W. (2018). English syllable structure. In O. Kang, R. Thomson, et J. Murphy (Eds.), The Routledge Handbook of Contemporary English Pronunciation (pp. 122-136). New York, NY: Routledge. 
Côté, M-H. (2005) Phonologie française. Manuscrit.

DeKeyser, R. (2003). Implicit and explicit learning. Dans C. Doughty et M. H. Long (Éds), The handbook of Second Language Acquisition (pp. 313-348). Malden, MA : Blackwell Pub.

Derwing, T. M. (2018). The role of phonological awareness in language learning. Dans The Routledge handbook of language awareness (1 ${ }^{\text {re }}$ éd., pp. 339-353). (S.1.) : Peter Garrett, Josep M. Cots.

Derwing, T. M., Munro, M. J., et Wiebe, G. (1998). Evidence in favor of a broad framework for pronunciation instruction. Language Learning, 48(3), 393-410. https://doi.org/10.1111/0023-8333.00047

Derwing, T. M., et Munro, M. J. (2005). Second language accent and pronunciation teaching: A research-based approach. TESOL Quarterly, 39(3), 379-397.

Eckman, F. R. (1977). Markedness and the contrastive analysis hypothesis. Language Learning, 27(2), 315-330.

Ellis, R., Loewen, S., et Erlam, R. (2006). Implicit and explicit corrective feedback and the acquisition of L2 grammar. Studies in Second Language Acquisition, 28(2). https://doi.org/10.1017/S0272263106060141

Field, J. (2005). Intelligibility and the listener: The role of lexical stress. Teachers of English to Speakers of Other Languages, Inc. (TESOL), 39(3), 399-423.

Flege, J. E. (1995). Second language speech learning: Theory, findings, and problems. Dans W. Strange (Ed.), Speech perception and linguistic experience: Issues in crosslanguage research (pp. 233-276). Timonium, MD: York Press.

Flege, J. E., Yeni-Komshian, G. H., et Liu, S. (1999). Age constraints on second-language acquisition. Journal of Memory and Language, 41(1), 78-104. doi :10.1006/jmla.1999.2638

Foote, J. A., Holtby, A. K., et Derwing, T. M. (2011). Survey of the teaching of pronunciation in adult ESL programs in Canada, 2010. Revue TESL du Canada, 29(1), 1-22.

Foote, J. A., Trofimovich, P., Collins, L., et Urzúa, F. S. (2016). Pronunciation teaching practices in communicative second language classes. The Language Learning Journal, 44(2), 181-196. https://doi.org/10.1080/09571736.2013.784345

Fox, A. (1990). The structure of German. Oxford : New York : Clarendon Press ; Oxford University Press.

Gut, U. (2009). Non-native speech: A corpus-based analysis of phonological and phonetic properties of L2 English and German. Frankfurt am Main; Oxford : Peter Lang. (OCLC: ocn359673616).

Hahn, L. (2004). Primary stress and intelligibility: Research to motivate the teaching of suprasegment. Teachers of English to Speakers of Other Languages, Inc. (TESOL), 38(2), 201-223.

Iverson, P., Kuhl, P. K., Akahane-Yamada, R., Diesch, E., Tohkura, Y. 'ich, Kettermann, A., et Siebert, C. (2003). A perceptual interference account of acquisition difficulties for non-native phonemes. Cognition, 87(1), B47-B57. doi :10.1016/S00100277(02)00198-1

Jenkins, J. (2002). A sociolinguistically based, empirically researched pronunciation syllabus for English as an international language. Applied Linguistics, 23(1), 83-103. 
John, P., et Cardoso, W. (2009). Francophone ESL learners' difficulties with English /h/. Dans B. Baptista, A. Rauber et M. Watkins (Eds.), Recent Research in Second Language Phonetics/Phonology: Perception and Production (pp. 118-140). Newcastle Upon Tyne: Cambridge Scholars Publishing.

Kemp, R. L. (2011). The perception of german dorsal fricatives by native speakers of English. Master thesis, University of Georgia. Cerca con Google. Repéré à https://getd.libs.uga.edu/pdfs/kemp_renee_1_201108_ma.pdf

Kennedy, S., Blanchet, J., et Trofimovich, P. (2014). Learner pronunciation, awareness, and instruction in French as a second language. Foreign Language Annals, 47(1), 79-96. https://doi.org/10.1111/flan.12066

Lee, J., Jang, J., et Plonsky, L. (2015). The effectiveness of second language pronunciation instruction: A meta-analysis. Applied Linguistics, 36(3), 345-366. doi :10.1093/applin/amu040

Levis, J. M. (2005). Changing contexts and shifting paradigms in pronunciation teaching. TESOL Quarterly, 39(3), 369. https://doi.org/10.2307/3588485

Loewen, S. (2015). Introduction to instructed second language acquisition. New York; London : Routledge, Taylor et Francis Group.

Mah, J., Goad, H., et Steinhauer, K. (2016). Using event-related brain potentials to assess perceptibility: The case of French speakers and English [h]. Frontiers in Psychology, 7. https://doi.org/10.3389/fpsyg.2016.01469

Morales Pech, D., et Izquierdo, J. (2011). L2 Phonology learnning among yong-adult learners of English: Effects of regular classroom-based instruction and L2 proficiency.

Munro, M. J., et Derwing, T. M. (2006). The functional load principle in ESL pronunciation instruction: An exploratory study. System, 34(4), 520-531. https://doi.org/10.1016/j.system.2006.09.004

Norris, J. M., et Ortega, L. (2000). Effectiveness of L2 instruction: A research synthesis and quantitative meta-analysis. Language Learning, 50(3), 417-528. https://doi.org/10.1111/0023-8333.00136

Saito, K., et Lyster, R. (2012). Effects of form-focused instruction and corrective feedback on L2 pronunciation development of / $/ \mathrm{/}$ by Japanese learners of English. Language Learning, 62(2), 595-633. doi :10.1111/j.1467-9922.2011.00639.x

Saito, K. (2015). Communicative focus on L2 phonetic form: Teaching Japanese learners to perceive and produce English / $\mathrm{I} /$ without explicit instruction. Applied Psycholinguistics, 36(2), 377-409.

Schiemann, E., et Bölck, M. (2008). hören - sprechen - richtig schreiben: Übungsprogramm zu Phonetik und Rechtschreibung für den Unterricht Deutsch als Fremdsprache. (2., durchges. Aufl). Stuttgart : Schmetterling Stuttgart.

Schmidt, R. (2010). Attention, awareness, and individual differences in language learning. Proceedings of CLaSIC 2010, 721-737.

Spada, N., et Tomita, Y. (2010). Interactions between type of instruction and type of language feature: A meta-analysis. Language Learning, 60(2), 263-308. https://doi.org/10.1111/j.1467-9922.2010.00562.x

Thomson, R. I., et Derwing, T. M. (2015). The effectiveness of L2 pronunciation instruction: A narrative review. Applied Linguistics, 36(3), 326-344. https://doi.org/10.1093/applin/amu076 
University of Iowa. (2014). Sounds of Speech. Sounds of Speech. [logiciel de phonétique]. Repéré à https://soundsofspeech.uiowa.edu/main/german 
Annexe A

Tâche de perception (prétest)

\begin{tabular}{|c|c|c|c|c|c|}
\hline & & \multicolumn{2}{|c|}{ À écouter (T1) } & oui & non \\
\hline 1 & /pf/ & Pfalz & Falz & X \\
\hline 2 & /ts/ & Weizen & weißen & & $\mathrm{X}$ \\
\hline 3 & - & spielen & spielen & $\mathrm{X}$ & \\
\hline 4 & $/$ ç/ & mich & misch & & $\mathrm{X}$ \\
\hline 5 & - & Dienstag & Dienstag & $\mathrm{X}$ & \\
\hline 6 & $/ \mathrm{h} /$ & heiß & Eis & & $\mathrm{X}$ \\
\hline 7 & $/ \mathrm{x} /$ & Buch & Stuhl & $\mathrm{X}$ & $\mathrm{X}$ \\
\hline 8 & - & Stuhl & Zeit & & $\mathrm{X}$ \\
\hline 9 & $/ \mathrm{ts} /$ & seit & finden & $\mathrm{X}$ & \\
\hline 10 & - & finden & Halt & & $\mathrm{X}$ \\
\hline 11 & $/ \mathrm{h} /$ & alt & Bahn & $\mathrm{X}$ & \\
\hline 12 & - & Bahn & Pfeile & & $\mathrm{X}$ \\
\hline 13 & $/ \mathrm{pf} /$ & Feile & machen & & $\mathrm{X}$ \\
\hline 14 & $/ \mathrm{X} /$ & Maschen & Kirche & & $\mathrm{X}$ \\
\hline 15 & $/ \mathrm{ç/}$ & Kirsche & & & \\
\hline
\end{tabular}


Annexe B

Tâche de perception (posttest)

\begin{tabular}{|c|c|c|c|c|c|}
\hline & & \multicolumn{2}{|c|}{ À écouter (T2) } & oui & non \\
\hline 1 & - & Bad & Bad & $\mathrm{X}$ & \\
\hline 2 & $/ \mathrm{h} /$ & aus & Haus & & $\mathrm{X}$ \\
\hline 3 & - & sauber & sauber & $\mathrm{X}$ & \\
\hline 4 & $/ \mathrm{pf} /$ & Pfau & «V» (fau) & & $\mathrm{X}$ \\
\hline 5 & $/ \mathrm{cc/}$ & Löcher & Löscher & & $\mathrm{X}$ \\
\hline 6 & $/ \mathrm{x} /$ & Tusch & Tuch & & $\mathrm{X}$ \\
\hline 7 & $/ \mathrm{ts} /$ & heißen & heizen & & $\mathrm{X}$ \\
\hline 8 & - & Flieger & Flieger & $\mathrm{X}$ & \\
\hline 9 & $/ \mathrm{h} /$ & helfen & Elfen & & $\mathrm{X}$ \\
\hline 10 & $/ \mathrm{ç/}$ & wischt & Wicht & & $\mathrm{X}$ \\
\hline 11 & - & Enkel & Enkel & $\mathrm{X}$ & \\
\hline 12 & $/ \mathrm{ts} /$ & Zinne & Sinne & & $\mathrm{X}$ \\
\hline 13 & - & Werbung & Werbung & $\mathrm{X}$ & \\
\hline 14 & $/ \mathrm{pf} /$ & fährt & Pferd & & $\mathrm{X}$ \\
\hline 15 & $/ \mathrm{x} /$ & wach & wasch & & $\mathrm{X}$ \\
\hline
\end{tabular}


Annexe C

Tâche de production (prétest)

\begin{tabular}{|c|c|}
\hline & Abend \\
\hline & März \\
\hline 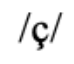 & Manchmal \\
\hline & $4+2-1=?$ \\
\hline & Pferd \\
\hline & Woche \\
\hline & Verhängt \\
\hline & $10 \div 5=?$ \\
\hline & Anruf \\
\hline & Nächte \\
\hline & Pfanne \\
\hline & $9-4=?$ \\
\hline & Haben \\
\hline$/ \mathrm{x} /$ & Kuchen \\
\hline & Ganze \\
\hline & $3 \times 4=$ ? \\
\hline$/ \mathrm{c}$ & Dich \\
\hline & Eltern \\
\hline$/ \mathrm{h} /$ & Hinter \\
\hline & $7+6=?$ \\
\hline$/ \mathbf{v} 1$ & Besucht \\
\hline & Pfund \\
\hline & Zeitung \\
\hline & $6 \div 2=?$ \\
\hline
\end{tabular}


Annexe D

Tâche de production (posttest)

\begin{tabular}{c|c} 
- & Wand \\
/ts/ & Zeitung \\
/ç/ & Dich \\
- & $4+1-4$ \\
/pf/ & Pfand \\
/x/ & Besucht \\
/h/ & Hinter \\
- & $12 / 14=$ \\
- & Leiter \\
/ç/ & Manchmal \\
/pf/ & Pferd \\
- & $8-3=$ \\
/h/ & Verhängt \\
/x/ & Woche \\
/ts/ & März \\
- & $2 \times 3=$ \\
/ç/ & Nächte \\
- & Drucker \\
/h/ & Haben \\
- & $3+2=$ \\
/x/ & Kuchen \\
/pf/ & Pfanne \\
/ts/ & Ganze \\
- & $8 / 8=$ \\
& \\
\hline
\end{tabular}

